\title{
Optical coherence tomography image for automatic classification of diabetic macular edema
}

\author{
PING WANG, JiA-Li LI*, Hao DING \\ Department of Electronics Information Engineering, School of Information Engineering, \\ Nanchang University, Nanchang 330031, China \\ *Corresponding author: lijiali@email.ncu.edu.cn
}

\begin{abstract}
Diabetic macular edema (DME) is the dominant reason of diabetic visual loss, so early detection and treatment of DME is of great significance for the treatment of diabetes. Based on transfer learning, an automatic classification method is proposed to distinguish DME images from normal images in optical coherence tomography (OCT) retinal fundus images. Features of the DME are automatically identified and extracted by the pre-trained convolutional neural network (CNN), which only involves fine-tuning the VGGNet-16 network without any user intervention. An accuracy of $97.9 \%$ and a sensitivity of $98.0 \%$ are acquired with the OCT images in the Duke data set from experimental results. The proposed method, a core part of an automated diagnosis system of the DME, revealed the ability of fine-tuning models to train non-medical images, allowing them can be classified with limited training data. Moreover, it can be developed to assist early diagnosis of the disease, effectively delaying (or avoiding) the progression of the disease, consequently.
\end{abstract}

Keywords: diabetic macular edema, optical coherence tomography, transfer learning, fine-tuning.

\section{Introduction}

With the high-speed development of medical imaging technology, medical image processing and analysis has entered the era of big data. Extracting useful knowledge from a large amount of medical image data provides more sufficient basis for the diagnosis and scientific research of clinical disease, and has become a research focus in academia and industry. The visibility of the internal structure of the retina makes it possible to diagnose a variety of human fundus diseases, including age-related macular degeneration (AMD) and diabetic macular edema (DME), which are among the most irreversible causes of vision loss in the elderly and diabetes, respectively [1]].

Optical coherence tomography (OCT) is an imaging technique that captures high -resolution three-dimensional images of biological tissue. In particular, complex spectral optical coherence tomography (CSOCT), produces images without parasitic mirror com- 

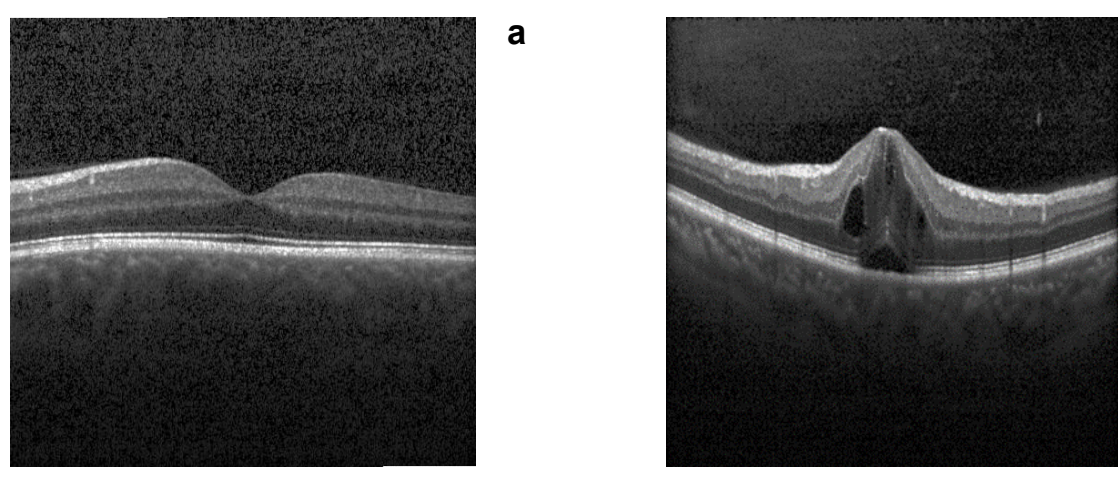

b

Fig. 1. Retinal OCT image of a subject: normal (a) and DME (b).

ponents [2]. One of its applications is to obtain retinal OCT volume, which can be employed to diagnose retinal diseases and treatment plans [ $\underline{3}]$. OCT image classification has also been investigated $[\underline{4}, \underline{5}]$. These findings have important implications for the use of OCT images automatically screening and the development of computer-aided diagnostic tool. The figure above is a retinal OCT image of a DME subject with geographically atrophic and fluid (Fig. 1b) and a retinal image of a normal subject (Fig. 1a).

It is shown that there are few reports on the application of DME screening based on deep learning algorithms. Some traditional image analyses demand manual development of a convolution matrix applied to images for edge detection and feature extraction. In recent studies, many computer automation systems implemented DME grading with image processing techniques using segmentation and foveal detection of retinal fundus image secretions [ $[\underline{6}, 7]$. Among them, NAYAK et al. presented a method relied on the detection and segmentation of macular exudates to detect and assess the severity of DME [6]. GIANCARDO et al. proposed an automatic scoring system in view of bleed probability map and wavelet decomposition []ㅡ. A method can be introduced by the extraction of morphological features: the location of the secretions was segmented with mathematical morphology to calculate the distance between the macular secretions and the center, applied to assess the severity of DME in each image, ultimately [9]. In addition, ZAIDI et al. developed a grading method to detect the location of secretions to assess the severity of DME, combining Gabor filtering, mathematical morphology, Otsu thresholds with Bayesian classifiers [10]. Based on matching correlation together with typical fovea characteristics, a fovea detection method is also proposed [11]. This algorithm was tested on some local data sets, and the sensitivity and specificity were up to 80.4 and $99.1 \%$, respectively. Furthermore, BABY and Chandy extracted the features in the wavelet subbands obtained by the double-tree complex wavelet transform (DT-CWT) for DME classification with Gaussian data description (GDD) [12]. МоокіAн et al. also proposed another method to extract image features based on high -order spectra to assess the severity of DME [7].

However, the performance of the scoring system described above relied on exudate segmentation, anatomical location, and feature extraction strategies. In order to change 
this limitation of traditional technology, deep learning was introduced. Deep learning technology is a new image classification technology with high precision [13]. The automatic feature extraction algorithm relying on deep learning has become a viable and effective method in some computer vision applications, such as ImageNet classification [14] and face recognition [15]. In the field of ophthalmology, some researchers have limited the application of deep learning to fundus photographs for automatic detection of diabetic retinopathy, visual field examination of glaucoma patients, grading of cataract, and segmentation of foveal microvessels [16, 17]. In fact, recent advances in image recognition and classification have turned to deep learning methods. LEE et al. developed a new algorithm based on convolutional neural network (CNN) to detect intraretinal fluid (IRF) on OCT images, and the results were consistent with those of clinicians [18]. A fully automatic retinal cyst segmentation technique was proposed for the OCT image stack obtained from commercial scanners, where the fast speckle denoising noise was calculated by bilateral filters while maintaining the boundary of the cystic macular edema (CME) [19]. A correlation-based feature subset (CFS) selection algorithm was proposed to screen the characteristics of OCT images based on linear configuration patterns, and to distinguish age-related macular degeneration (AMD), diabetic macular edema (DME) and healthy macula [20].

A common phenomenon, requiring a large amount of data to increase the generalization ability of the training model in machine learning and deep learning, has been discovered. In the case of insufficient data volume, models are usually easy to overfitting, which will have a negative impact on performance during testing. In general, researchers build models with data-driven feature quantifiers (filters), i.e., a higher order model, to prevent overfitting. Therefore, to compensate the imperfections of classical machine learning, a transfer learning [21] has been introduced, in which the model is trained on tasks with sufficient data, and then the model parameters fine-tuned used for the required target task. In this paper, an automatic diagnostic system by fine-tuning the VGGNet-16 network is proposed to distinguish DME images from optical coherence tomography (OCT) retinal fundus normal images.

The rest of this paper is organized as follows. In Section 2, the experimental method is given as model theoretical fundamentals. Then the proposed image classification algorithm for diabetes macular edema based on transfer learning is described in detail and some evaluation indicators are introduced. Next in Section 3, the comparison of experimental performance between different methods on the same data set is discussed and the experimental results are shown. Finally, some concluding remarks are drawn in the last section.

\section{Experimental method}

\subsection{Experimental platform and data set}

The deep neural network of this experiment is built on the TensorFlow deep learning framework, and uses the Python-based machine learning tool (scikit-learn) to interact 

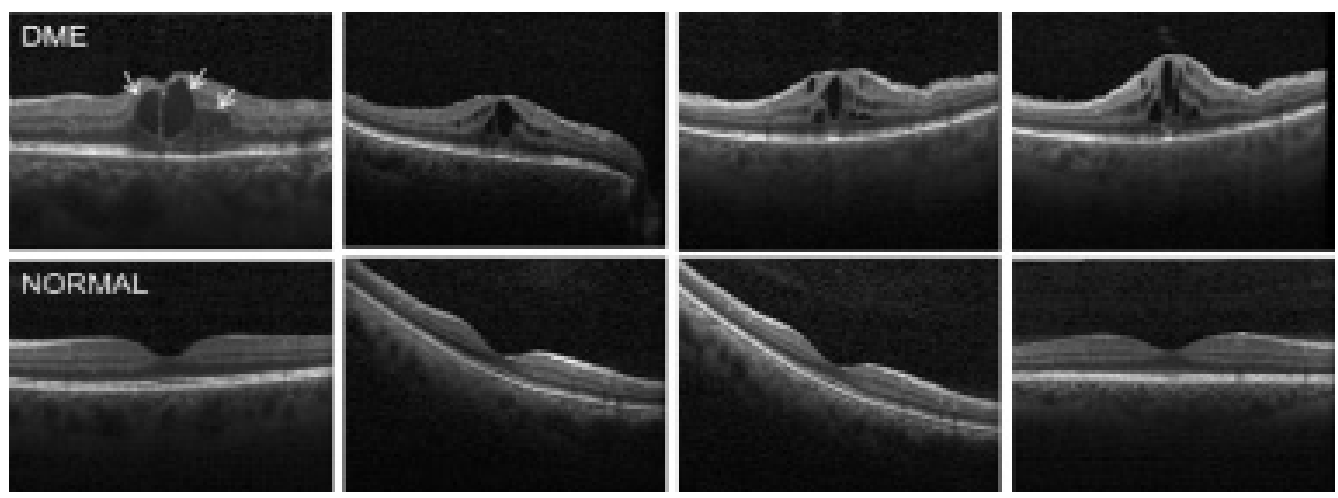

Fig. 2. Representative OCT image.

with TensorFlow's Python interface. The experimental hardware platform includes: Intel Core i5 7500k processor, 8 GB memory, graphics card for NVIDIA GeForce GTX1060, 6 GB memory.

The dataset (Duke data set) required for this study evaluation is a publicly available OCT data set from Duke University [22]. Duke data set consists of 45 subjects (15 dry AMD, 15 DME, and 15 normal) and each with multiple images (example as shown in Fig. 2). DME images and normal ones are reconstructed into a new data set for this experimental study. However, some images could not provide a clear view of the retina because of imaging techniques, such as some unclear illumination and motion blur images, which were excluded. The final OCT image dataset consists of $522 \mathrm{DME}$ images and 1398 normal images.

\subsection{Transfer learning algorithm design}

An effective transfer learning algorithm to process medical images should be developed to provide accurate and timely diagnosis of key pathologies in each image. Transfer learning is a very impressive technique, especially under limited data. The optimized lower-level weights are fine-tuned by a feedforward network to identify the structure in the general image, and the upper layer's weights are retrained by backpropagation instead of training a completely blank network. The model obtained can distinguish features of a particular category of images (e.g. images of the eye), not only faster but with fewer training examples and less calculate ability [23]. Figure 3 is a schematic of transfer learning.

The schematic depicts that a convolutional neural network trained on 1000 categories of ImageNet data sets can be adapted to improve the accuracy of training the network on new data sets of eye images and shorten the training duration.

The proposed algorithm pre-initializes the VGGNet-16 model parameters with the trained model on ImageNet, fixes the shallow network parameters without updating, and then fine-tunes the deep network parameters, while the final, fully connected layers will be re-established. The comprehensive research includes: 

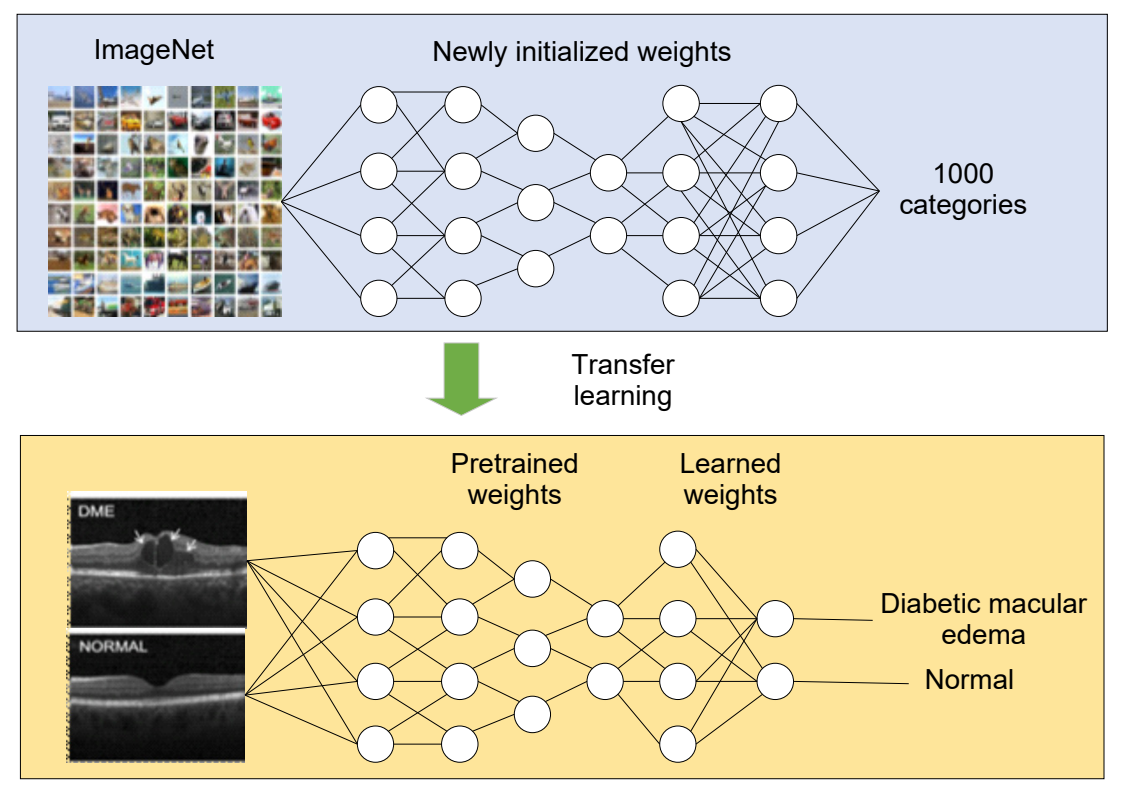

Fig. 3. Schematic diagram of transfer learning.

1) Pre-processing: adjusting the OCT images to the pixel size required by the VGGNet-16 network, and then dealing with the data distribution unbalance problem with the oversampling strategy;

2) Transfer learning: since the CNN model has a huge number of parameters, its operation process is time-consuming, leading to excessive fitting easily. Therefore, the VGGNet-16 network parameters are pre-initialized with the transfer learning method;

3) Classifier design: extracting the OCT images features of network learning is applied in the last connection layer, meanwhile image features are automatically classified with the softmax function;

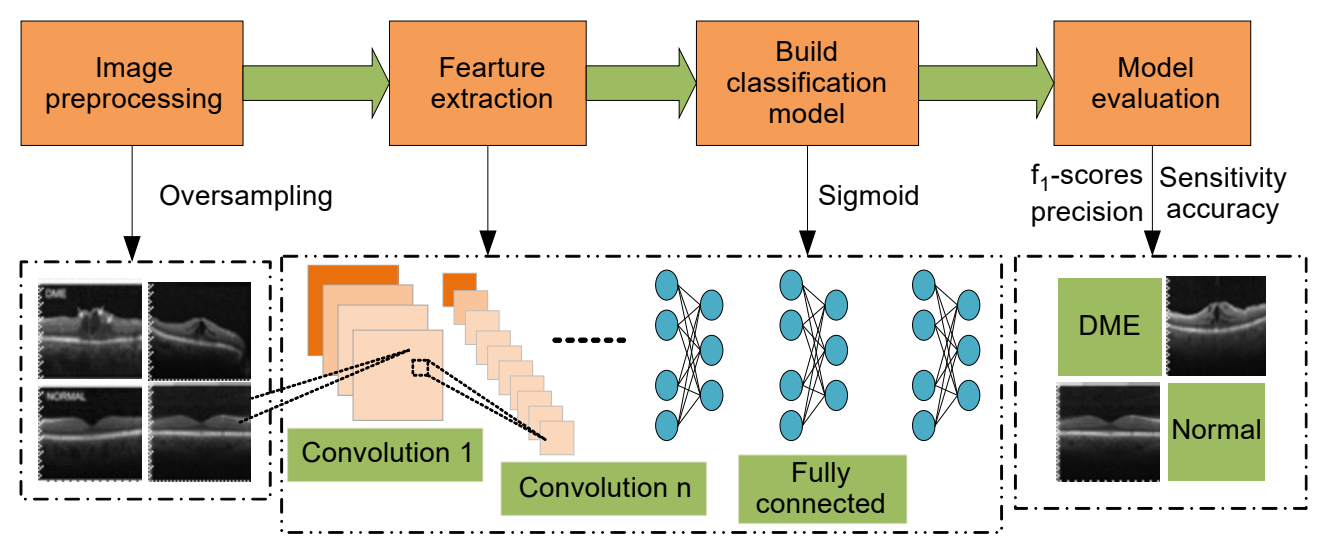

Fig. 4. System block diagram. 
4) Model evaluation: the classification results are evaluated by a whole string of evaluation indicators (e.g. sensitivity, accuracy, confusion matrix) to verify the performance of the model. Figure 4 shown system block diagram.

\subsection{VGGNet16 network structure}

In Keras, the application module comprises an auxiliary Keras application, which is provided with Keras model pre-trained weights. One can use these models directly or modify the model to complete transfer learning.

VGGNet-16 ranked second in the Image-Net competition and ranked first in positioning in 2014 [24], which is one of CNN's classic architectures, and widely used in the classification of other medical images. VGGNet- 16 consists of 13 convolutional layers with 3 fully connected layers and the size of the input image is $224 \times 224 \times 3$. Each convolution layer is followed by an activation layer containing the ReLU activation function, which is then connected to the pooling layer. The convolutional layer of VGGNet-16 with $3 \times 3 \times 3$ convolution kernels reduces the number of training parameters and increases that of the convolution kernels compared with the convolution kernel of AlexNet network [25].

The VGGNet-16 model and its simple modifications will be exploited. As shown in Fig. 5, the input of the first fully connected layer is $7 \times 7 \times 512$ and the output is a one-dimensional vector of 12800 . The first fully connected layer is equivalently represented by an effective convolutional layer with a convolution kernel size of $5 \times 5$ and a stride of 1 . The output is $1 \times 1 \times 12800$, equivalent to the full connection layer, which can more effectively predict the sliding window of the test image. After that, a flatten layer between the replaced convolutional layer and the second fully connected

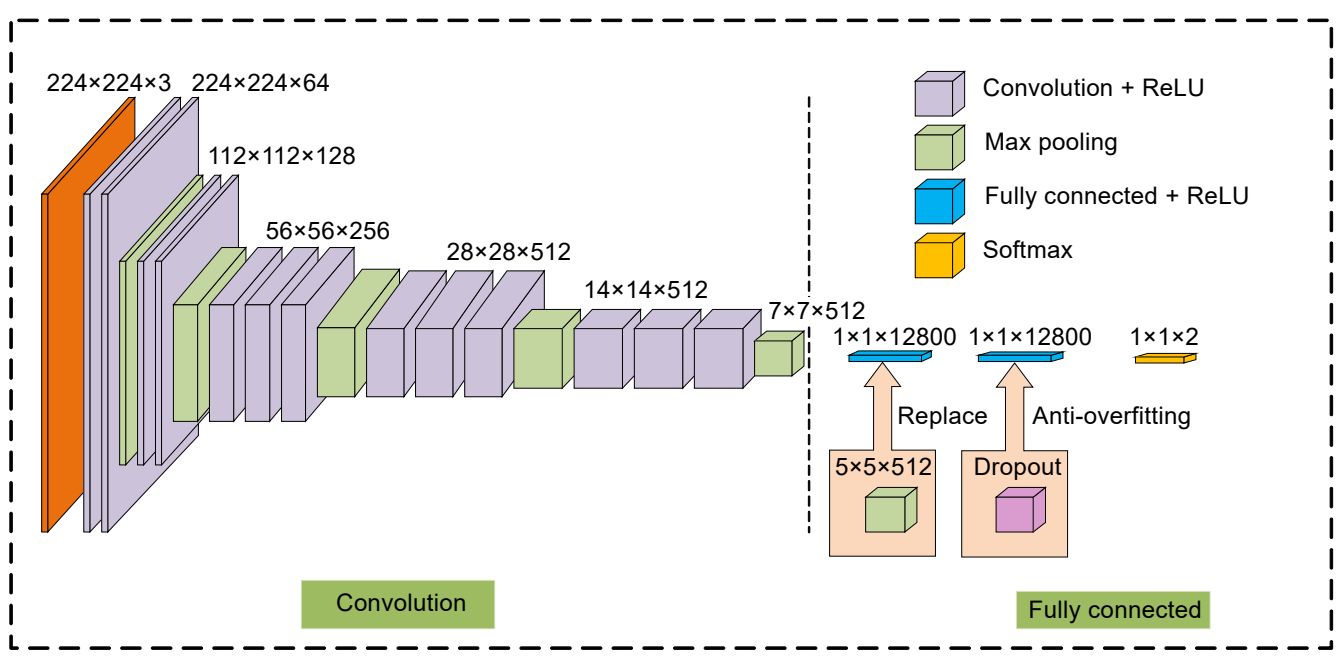

Fig. 5. VGGNet-16 structure diagram. 
layer makes the multidimensional input one-dimensional. Next, the dropout layer is appended to the second fully connected layer, which reduces the over-fitting phenomenon by weakening the interaction among the features due to too small data volume. The third fully-connected layer, a dense layer, is activated with softmax and the binary cross entropy is considered as a loss function. Finally, after three fully connected layers, we get a binary result: 0 (normal) or 1 (DME).

\subsection{Evaluation indicators}

From a clinical perspective, DME automatic detection focuses more on the accuracy of target detection and the diversity of methods, and does not care about the specific details of the methods. For detailed statistical evaluation, the performance of the proposed system is measured with sensitivity, precision, accuracy and $f_{1}$-scores as figures of merit. Therefore, the following indicators from the image level are used, respectively,

$$
\begin{aligned}
& R=\mathrm{SE}=\frac{T_{\mathrm{P}}}{T_{\mathrm{P}}-T_{\mathrm{N}}} \\
& \mathrm{AC}=\frac{T_{\mathrm{P}}+T_{\mathrm{N}}}{T_{\mathrm{P}}+T_{\mathrm{N}}+F_{\mathrm{P}}+F_{\mathrm{N}}} \\
& P=\frac{T_{\mathrm{P}}}{T_{\mathrm{P}}+F_{\mathrm{P}}} \\
& f_{1} \text {-score }=\frac{2 P R}{P+R}
\end{aligned}
$$

where $T_{\mathrm{P}}\left(T_{\mathrm{N}}\right)$ is true positive (negative), indicating that the real category of the sample is a positive (negative) class, but the result of the model recognition is a positive (negative) class. $F_{\mathrm{P}}\left(F_{\mathrm{N}}\right)$ is false positive (negative), which means the real category of the sample is a negative (positive) class, but the result of model recognition is a positive (negative) class. SE, i.e., sensitivity, reflects the probability that normal images will not be missed. AC, i.e., accuracy, is the proportion of all judgments correctly obtained as a percentage of the total. The positive category is normal images and the negative category is DME images. $P$, i.e., precision of other indicators, indicates the proportion of samples that are truly positive in the sample identified as positive and the higher the precision, the better the effect of the model, normally. $R$, i.e., recall rate, equals the sensitivity index. That is to say, the model correctly identifies the ratio of the number of positive samples to the total number of positive samples. In general, the higher the recall rate, the more positive samples are predicted correctly by the model. At the same time, the performance of model is of higher quality. And $f_{1}$-score, i.e., the balanced 
score, is defined as the harmonic mean of the correct rate and the recall rate. The receiver operating characteristics (ROC) and area under curve (AUC) are also employed to evaluate the parameter-independent classification performances.

\section{Experimental results and discussion}

The OCT image data set consists of 522 DME images and 1398 normal images. The raw data was split into "test sets" and "training sets" by using the train_test_split module in sklearn.cross_validation. The test sets account for $20 \%$ of the total set, remaining $80 \%$ constitute the training sets and the seed of the fixed random number is guaranteed to obtain the same random number for each division. During model training, the randomness caused by model fine-tuning produces variable experimental results. Therefore, this study repeated experiments and saved the best model (97.9\%). After 50 epochs experiments, we show sensitivity, precision, $f_{1}$-score, accuracy and AUC obtained by our proposed method in Table 1.

In the field of machine learning, the confusion matrix is a visual display tool to evaluate the quality of a classification model. Each column of the matrix represents the sample condition of the model prediction, and each row of the matrix represents the real situation of the sample. The confusion matrix is another method to judge the degree of the classification beside ROC and AUC.

The prediction of each category can be obtained from the confusion matrix in Fig. $6 \mathbf{a}$. We randomly selected 98 images (49 DME, 49 normal) from the original data set for

$\mathrm{T}$ a b l e 1. Quantitative results on the test set.

\begin{tabular}{llllll}
\hline Class & Precision & Sensitivity & $f_{1}$-score & Accuracy & AUC \\
\hline DME & 1.00 & 0.960 & 0.982 & 0.978 & 0.980 \\
Normal & 0.970 & 1.00 & 0.982 & 0.981 & 0.980 \\
Average & 0.985 & 0.980 & 0.982 & 0.979 & 0.980 \\
\hline
\end{tabular}

a

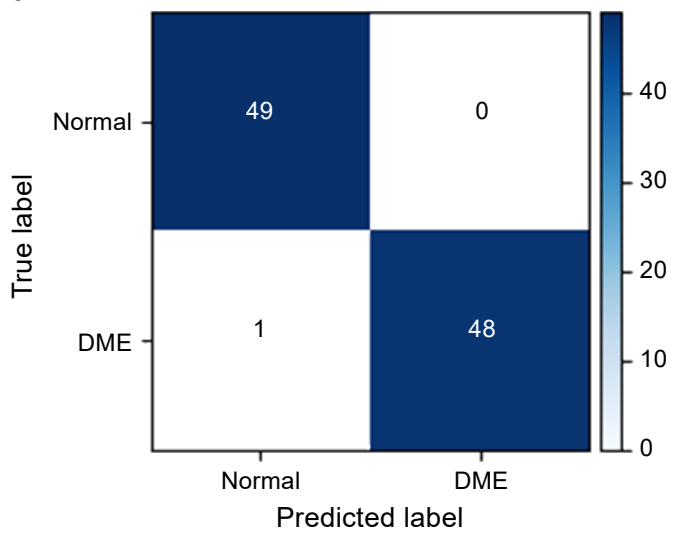

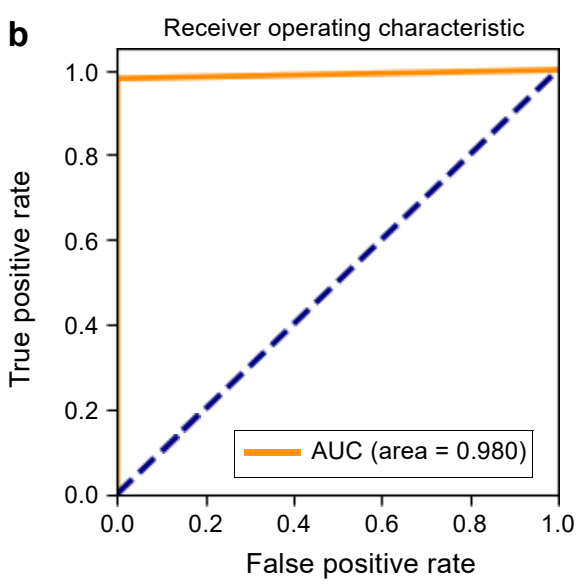

Fig. 6. Confusion table (a), and receive operating characteristic (ROC), AUC $=0.980$ (b). 
T a b l e 2. Performance comparison on the same data set.

\begin{tabular}{|c|c|c|c|c|}
\hline Method & Sensitivity & $f_{1}$-score & Accuracy & AUC \\
\hline Ref. [르] & 1.000 & 0.937 & 0.933 & NA \\
\hline Ref. [26] & 0.714 & 0.704 & 0.700 & NA \\
\hline Ref. [27] & 0.866 & 0.928 & 0.933 & NA \\
\hline Ref. [28] & 0.800 & 0.888 & 0.900 & NA \\
\hline Proposed & 0.980 & 0.982 & 0.979 & 0.980 \\
\hline
\end{tabular}

an experimental evaluation. We found that the accuracy and precision of the experiment reached $98.9 \%$ and $98 \%$, respectively. In the case where the true value is positive (normal), the correct proportion of prediction is $100 \%$ (sensitivity). In addition, in the case where the true value is negative (DME), the correct proportion of prediction is $97.9 \%$ (specificity). While it is claimed that our method is not necessarily a better method than the others, but it is clear that our model performs well in terms of the ROC and AUC value (Fig. 6b).

To verify the reliability of this study, Table 2 shows the comparison between different methods of sensitivity, $f_{1}$-score, accuracy and AUC values on the same data set (Duke data set). An area (AUC) under the receiver operator characteristics curve value was not reported by these researches which is why the corresponding table columns in Table 2 contain "NA". In the following paragraph, we explain the results of each method in details.

On the one hand, the sensitivity of SRINIVASAN et al. [22] achieves $100 \%$ far more than Venhuizen et al. [26], Lemaître et al. [27] and SidibÉ et al. [228], and compared to these three methods, our proposed method implements a sensitivity of $98.0 \%$. On the other hand, in terms of another important metric for the performance, the measurement is $f_{1}$-score, the highest achieved $98.2 \%$ by our proposed method and the lowest value (70.4\%), obtained by VENHUIZEN et al. The proposed method achieved the highest value in both $f_{1}$-score and accuracy $(97.9 \%)$, which is higher than other methods, with the second highest value (93.3\%) obtained by SRINIVASAN et al. and LeMAÎTRE et al. In other words, the performance of the proposed method is better in these performance metrics.

\section{Conclusion}

Medically, the image examination of eye diseases mainly remains at the doctor's manual diagnosis stage, but manual diagnosis could bring about high labor costs. In this paper, a deep convolutional neural network is used to achieve automatic classification of diabetic macular edema by analyzing the characteristics of the patient's OCT images of the eye. However, in order to solve the problem that the model commonly used in the classification algorithm of diabetic macular edema based on convolutional neural network is difficult to train and easy to overfit, this paper proposed an image classification algorithm based on transfer learning and softmax classifier. Unfortunately, the current study mainly focused on classifying the pathological condition, and there was no detection of its severity which is very important in clinical set up. In the future, we 
hope to predict images containing severity levels and expand the current framework to detect other abnormal structures, namely vitreous macular traction, retinal anterior membrane, drusen and other eye diseases, such as age-related macular degeneration.

Acknowledgments - This work is supported by the Jiangxi Outstanding Youth Fund Project (Grant No. 2018ACB21038), Jiangxi Science and Technology Department Science and Technology Support Program (Grant No. 20151BBG70057), Jiangxi Provincial Department of Education Science and Technology Funding Project (Grant No. GJJ14137) and the National Natural Science Foundation of China (Grant No. 61463035).

\section{References}

[1] Bowes Rickman C., Farsiu S., Toth C.A., Klingeborn M., Dry age-related macular degeneration: mechanisms, therapeutic targets, and imaging, Investigative Ophthalmology \& Visual Science 54(14), 2013, pp. ORSF68-ORSF80, DOI: 10.1167/iovs.13-12757.

[2] Szkulmowski M., Bajraszewski T., Szkulmowska A., Targowski P., Kowalczyk A., Efficient residual error reduction in complex spectral optical coherence tomography with arbitrary or unknown phase, Optica Applicata 36(1), 2006, pp. 147-155.

[3] Fujimoto J.G., Drexler W., Schuman J.S., Hitzenberger C.K., ISP focus issue: optical coherence tomography (OCT) in ophthalmology, Optics Express 17(5), 2009, pp. 3978-3979, DOI: 10.1364/ OE.17.003978.

[4] Garcia-Allende P.B., Amygdalos I., Dhanapala H., Goldin R.D., Hanna G.B., Elson D.S., Morphological analysis of optical coherence tomography images for automated classification of gastrointestinal tissues, Biomedical Optics Express 2(10), 2011, pp. 2821-2836, DOI: 10.1364/ BOE.2.002821.

[5] Gossage K.W., TKaczyk T.S., Rodriguez J.J., Barton J.K., Texture analysis of optical coherence tomography images: feasibility for tissue classification, Journal of Biomedical Optics 8(3), 2003, pp. 570-575, DOI: $10.1117 / 1.1577575$.

[6] NAyAk J., Bhat P.S., Acharya U.R., Automatic identification of diabetic maculopathy stages using fundus images, Journal of Medical Engineering \& Technology 33(2), 2009, pp.119-129, DOI: $10.1080 / 03091900701349602$.

[7] Mookiah M.R.K., Acharya U.R., Chandran V., Martis R.J., Tan J.H., Koh J.E.W., Chua C.K., Tong L., LAUde A., Application of higher-order spectra for automated grading of diabetic maculopathy, Medical \& Biological Engineering \& Computing 53(12), 2015, pp. 1319-1331, DOI: $10.1007 / \mathrm{s} 11517-015-1278-7$.

[8] Giancardo L., Meriaudeau F., Karnowski T.P., Li Y., Garg S., Tobin K.W., Chaum E., Exudate -based diabetic macular edema detection in fundus images using publicly available datasets, Medical Image Analysis 16(1), 2012, pp. 216-226, DOI: 10.1016/j.media.2011.07.004.

[9] Tariq A., Akram M.U., Shaukat A., Khan S.A., Automated detection and grading of diabetic maculopathy in digital retinal images, Journal of Digital Imaging 26(4), 2013, pp. 803-812, DOI: $10.1007 /$ s10278-012-9549-4.

[10] ZAIDI Z.Y., AKRAM M.U., TARIQ A., Retinal image analysis for diagnosis of macular edema using digital fundus images, [In] 2013 IEEE Jordan Conference on Applied Electrical Engineering and Computing Technologies (AEECT), Amman, 2013, pp. 1-5, DOI: 10.1109/AEECT.2013.6716476.

[11] Sinthanayothin C., Boyce J.F., Cook H.L., Williamson T.H., Automated localisation of the optic disc, fovea, and retinal blood vessels from digital colour fundus images, British Journal of Ophthalmology 83(8), 1999, pp. 902-910, DOI: 10.1136/bjo.83.8.902.

[12] Baby C.G., Chandy D.A., Content-based retinal image retrieval using dual-tree complex wavelet transform, [In] 2013 International Conference on Signal Processing, Image Processing \& Pattern Recognition, Coimbatore, 2013, pp. 195-199, DOI: 10.1109/ICSIPR.2013.6497987. 
[13] Litjens G., Kooi T., Bejnordi B.E., Setio A.A.A., Ciompi F., Ghafoorian M., van der LaAk J.A.W.M., GinNEKEn B., SÁNCHez C.I., A survey on deep learning in medical image analysis, Medical Image Analysis 42, 2017, pp. 60-88, DOI: 10.1016/j.media.2017.07.005.

[14] Krizhevsky A., Sutskever I., Hinton G., ImageNet classification with deep convolutional neural networks, Advances in Neural Information Processing Systems 25(2), 2012, pp. 1097-1105.

[15] Sun Y., LiAng D., WANG X., TANG X., DeepID3: face recognition with very deep neural networks, eprint arXiv:1502.00873, February 2015.

[16] Asaoka R., Murata H., Iwase A., Araie M., Detecting preperimetric glaucoma with standard automated perimetry using a deep learning classifier, Ophthalmology 123(9), 2016, pp. 1974-1980, DOI: 10.1016/j.ophtha.2016.05.029.

[17] Prentašić P., Heisler M., Mammo Z., Lee S., Merkur A., Navajas E., Beg M.F., Šarunic M., LONČARIĆ S., Segmentation of the foveal microvasculature using deep learning networks, Journal of Biomedical Optics 21(7), 2016, article 075008, DOI: 10.1117/1.JBO.21.7.075008.

[18] Lee C.S., Tyring A.J., Deruyter N.P., Wu Y., Rokem A., Lee A.Y., Deep-learning based, automated segmentation of macular edema in optical coherence tomography, Biomedical Optics Express 8(7), 2017, pp. 3440-3448, DOI: 10.1364/BOE.8.003440.

[19] Wilkins G.R., Houghton O.M., Oldenburg A.L., Automated segmentation of intraretinal cystoid fluid in optical coherence tomography, IEEE Transactions on Biomedical Engineering 59(4), 2012, pp. 1109-1114, DOI: 10.1109/TBME.2012.2184759.

[20] Wang Y., Zhang Y., Yao Z., Zhao R., Zhou F., Machine learning based detection of age-related macular degeneration (AMD) and diabetic macular edema (DME) from optical coherence tomography (OCT) images, Biomedical Optics Express 7(12), 2016, pp. 4928-4940, DOI: 10.1364/BOE.7.004928.

[21] Ding S., Zhu H., Jia W., Su C., A survey on feature extraction for pattern recognition, Artificial Intelligence Review 37(3), 2012, pp. 169-180, DOI: 10.1007/s10462-011-9225-y.

[22] Srinivasan P.P., Kim L.A., Mettu P.S., Cousins S.W., Comer G.M., Izatt J.A., Farsiu S., Fully automated detection of diabetic macular edema and dry age-related macular degeneration from optical coherence tomography images, Biomedical Optics Express 5(10), 2014, pp. 3568-3577, DOI: 10.1364/BOE.5.003568.

[23] Pan S.J., Yang Q., A survey on transfer learning, IEEE Transactions on Knowledge and Data Engineering 22(10), 2010, pp. 1345-1359, DOI: 10.1109/TKDE.2009.191.

[24] Simonyan K., Zisserman A., Very Deep Convolutional Networks for Large-Scale Image Recognition, arXiv:1409.1556, 2014.

[25] Rastegari M., Ordonez V., Redmon J., Farhadi A., XNOR-Net: ImageNet classification using binary convolutional neural networks, [In] Computer Vision - ECCV 2016, Leibe B., Matas J., Sebe N., Welling M. [Eds.], Lecture Notes in Computer Science, Vol. 9908, Springer, Cham, 2016, pp. 525 -542, DOI: 10.1007/978-3-319-46493-0_32.

[26] Venhuizen F.G., van Ginneken B., Bloemen B., van Grinsven M.J.J.P., Philipsen R., Hoyng C., THEelen T., SÁnChez C.I., Automated age-related macular degeneration classification in OCT using unsupervised feature learning, Proceedings of SPIE 9414, 2015, article 94141I, DOI: 10.1117 12.2081521.

[27] Lemaître G., Rastgoo M., Massich J., Cheung C.Y., Wong T.Y., Lamoureux E., Milea D., MÉRIAUdeAu F., SidiBÉ D., Classification of SD-OCT volumes using local binary patterns: experimental validation for DME detection, Journal of Ophthalmology, 2016, article 3298606, DOI: $10.1155 / 2016 / 3298606$.

[28] Sidibé D., Sankar S., Lemaître G., Rastgoo M., Massich J., Cheung C.Y., Tan G.S.W., Milea D., Lamoureux E., Wong T.Y., Mériaudeau F., An anomaly detection approach for the identification of DME patients using spectral domain optical coherence tomography images, Computer Methods and Programs in Biomedicine 139, 2017, pp. 109-117, DOI: 10.1016/j.cmpb.2016.11.001. 